\title{
Microstructure and phase stress partition of Mo fiber reinforced CuZnAl composite
}

Feng Yang ${ }^{1,2}$, Dingrui $\mathrm{Ni}^{3}$, shijie hao ${ }^{1,2}$, Sirui $\mathrm{Li}^{2}$, Zongyi $\mathrm{Ma}^{3}$, Yinong $\mathrm{Liu}^{4}$, Chun Feng ${ }^{5}$, Lishan Cui ${ }^{1,2 a)}$

${ }^{1}$ State Key Laboratory of Heavy Oil Processing, China University of Petroleum-Beijing, Beijing 102249, China

${ }^{2}$ Department of Materials Science and Engineering, China University of Petroleum-Beijing, Beijing 102249, China

${ }^{3}$ Shenyang National Laboratory for Materials Science, Institute of Metal Research, Chinese Academy of Sciences, 72 Wenhua Road, Shenyang 110016, China

${ }^{4}$ School of Mechanical and Chemical Engineering, The University of Western Australia, Crawley, WA 6009, Australia

${ }^{5}$ Department of Materials Physics and Chemistry, University of Science and Technology Beijing, Beijing 100083, China

\begin{abstract}
A Mo fiber reinforced CuZnAl composite was prepared by means of friction stir processing and wire drawing. Reinforcing effect of the Mo fiber and phase stress partition in the composite were investigated by means of in-situ synchrotron X-ray diffraction. The maximum elastic strain of the Mo fiber achieved was $1.8 \%$, implying a component stress of $550 \mathrm{MPa}$ on the fibers. The Mo fibers, with a volume fraction of $10 \%$, carried $80 \%$ of stress fraction during tensile deformation. The change of modulus caused by stress-induced martensitic transformation strain resulted in redistribution of the phase stress partition between Mo fibers and CuZnAl matrix.
\end{abstract}

Keywords: fiber reinforced composites; synchrotron; shape memory alloys; mechanical properties; CuZnAl

\section{Introduction}

CuZnAl shape memory alloy (SMA) is considered an economical alternative to NiTi SMA due to its lower cost. However, its mechanical properties are poor compared to those of NiTi SMA, in particular yield strength. Much effort has been made in the past two decades to improve the mechanical properties of CuZnAl, for example by adding $\mathrm{Zr}, \mathrm{Ti}, \mathrm{V}$ and $\mathrm{Cr}$ [1-4], but the results achieved to date have been less than satisfactory.

Fiber reinforced metal matrix composite has attracted great attention. It is known that metallic fibers generally have exceptional mechanical properties than their bulk counterparts, which is considered to be one of promising reinforcements $[5,6]$. In the past twenty years, many attempts have been made to create bulk composite of superior strength using nano and 
micro-sized metallic and ceramic fibers. However, these studies [7-9] have proven to be unsuccessful in that the exceptional intrinsic mechanical properties cannot be achieved in conventional metal matrices deforming by dislocation slip, as is understood now. Recently, Hao et al reported that $\mathrm{Nb}$ nanowires embedded in a NiTi SMA matrix [10,11] deforming by stress-induced martensitic transformation (SIMT) can exhibit ultra-large elastic strains up to $6.4 \%$, which rendered the composite extraordinary mechanical properties. They proposed that the matrix should deform via SIMT, and the exceptional mechanical properties of the fiber can be inherited in composite.

CuZnAl SMA also deforms by stress-induced martensitic transformation similar to that of NiTi SMA. Therefore, it may be expected that a CuZnAl matrix may also be able to induce large elastic strains in embedded metal fibers, thus high load sharing by the fibers sustained. Moreover, it is known that the stress sustained by the embedded nanowires is related to the elastic strain and proportional to its elastic modulus [11]. Thus, it can be conjured that the composite made with CuZnAl SMA and the metal fibers with high elastic modulus may exhibit superior mechanical properties. However, the conventional metals with high elastic modulus usually have a high melting point. Corresponding metal fibers are difficult to be prepared by melting and wire drawing.

In this work, we prepared a Mo fiber reinforced CuZnAl composite by friction stir processing (FSP) and wire drawing. Mo was chosen for its high Young's modulus. Compare to pure CuZnAl, Mo-CuZnAl composites have higher strength and appropriate plasticity. Reinforcing effect of Mo fiber was investigated by in situ synchrotron x-ray diffraction during tensile loading. The elastic strain of Mo fiber is about $1.8 \%$, which carried $80 \%$ of stress fraction during tensile deformation. The phase stress partition in composites was discussed. The stress fraction carried by the reinforcement and the matrix in dislocation slip matrix composites increases or keeps almost constant with increasing macroscopic strain. However, the change of modulus caused by SIMT strain resulted in redistribution of the phase stress partition between Mo fibers and CuZnAl matrix.

\section{Experimental}

A molybdenum powder (average particale diameter $3 \mu \mathrm{m}$ ) was first incorporated into Cu-25.79 wt\% Zn-4.62 wt\% Al sheets (thickness 8mm) by FSP. FSP was performed using a tool that consisted of a flat, scrolled $24 \mathrm{~mm}$ diameter shoulder with a threaded $8 \mathrm{~mm}$ diameter pin that was approximately $4.5 \mathrm{~mm}$ in length. A four-pass FSP, with a $100 \%$ overlap and the same forward directions, was conducted at a rotational rate of $600 \mathrm{rpm}$ and a traversing speed of $100 \mathrm{~mm} / \mathrm{min}$. The obtained CuZnAl composite sheets reinforced by Mo particles were rolled into a sheet of $2 \mathrm{~mm}$ thickness. The sheet was then cut into $2 \times 2 \mathrm{~mm}$ strips by means of electrical discharge machining. These strips were cold drawn into wires of $0.28 \mathrm{~mm}$ in diameter with $630^{\circ} \mathrm{C}$ for 10 minutes intermediate anneals. The final anneal was at $800^{\circ} \mathrm{C}$ for 10 minutes and then quenched in water. This processing reduced the Mo particles into Mo fibers all aligned along the wire axial direction. 
The microstructures were observed with scanning electron microscopy (SEM) using a FEI Quanta 200F SEM and transmission electron microscopy (TEM) using a JEM 2100 TEM operating at $200 \mathrm{kV}$. The TEM specimens were prepared by mechanically grinding to a thickness of $30 \mu \mathrm{m}$ and then milling using Gatan 691 ion milling instrument. X-ray diffraction (XRD) measurements were performed using the diffractometer on Bruker D8 Focus oprerating at $40 \mathrm{kV}$ and $40 \mathrm{~mA}$ with $\mathrm{Cu} \mathrm{k}_{\alpha}$ radiation.

Deformation behaviour of the Mo fibers embedded in the CuZnAl matrix was studied by means of in situ synchrotron x-ray diffraction during tensile deformation. The measurements were performed at the 11-ID-C beamline of the Advanced Photon Source, Argonne National Laboratory. High energy x-rays having a beam size of $0.4 \times 0.4 \mathrm{~mm}$ and a wavelength of $0.10798 \AA$ were used to obtain two-dimensional (2-D) diffraction patterns in the transmission geometry.

\section{Results and discussions}

Figure 1 shows scanning electron microscope (SEM) investigations of Mo fiber reinforced CuZnAl composite Figure 1a shows an SEM micrograph of the transverse section of the composite wire. Mo appears as ribbons of 50-150nm in thickness and 500nm in width. Figure $1 \mathrm{~b}$ shows the morphology of the longitudinal section of the composites. Mo fibers are dispersed and well aligned in the CuZnAl matrices along the wire axis. The volume fraction of the Mo fibers is about $10 \%$ measured by sectional area measurement, the rest is CuZnAl matrix. The microstructure of composite was further observed by using of the transmission electron microscope (TEM). Figure 1c shows a TEM micrograph of the composite. Figure 1d shows the corresponding select area electron diffraction (SAED) pattern of area A. The diffraction pattern is indexed to the $\beta$ phase of CuZnAl. Figure 1e shows the corresponding SAED Pattern of area B. The pattern is fully indexed to the 9R monoclinic martensite with lattice parameters $\mathrm{a}=0.457 \mathrm{~nm}, \mathrm{~b}=0.262 \mathrm{~nm}, \mathrm{c}=1.943 \mathrm{~nm}$. Mo fibers are surrounded by martensites and $\beta$ phase in Figure 1c.
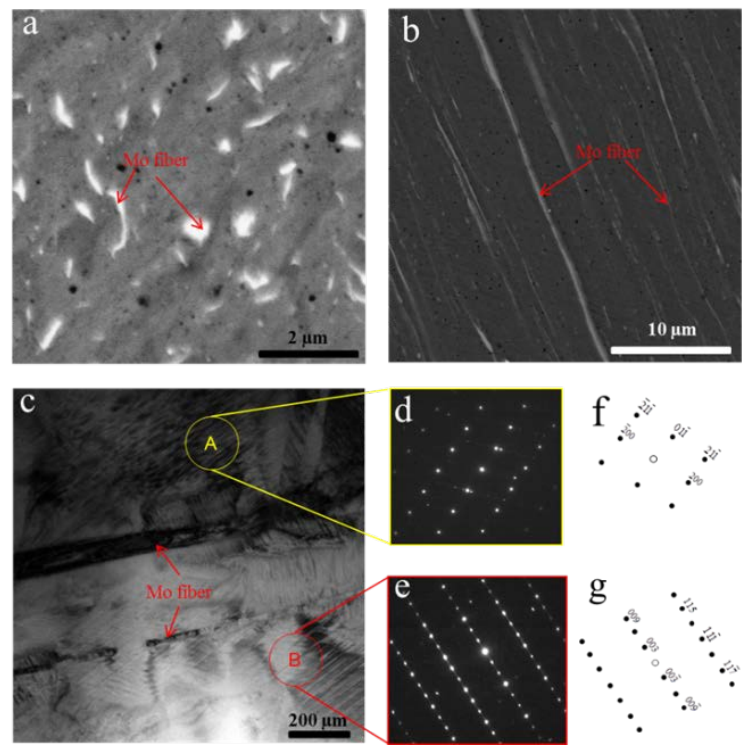
Figure 1. a. SEM image of transverse section of Mo fiber reinforced CuZnAl composites, b. SEM image of longitudinal section of composites, c. TEM image of the composite, d. corresponding SADP of area A, e. corresponding SADP of area B, f. indexed pattern in d, $g$ indexed pattern in e.

Figure 2a shows the XRD pattern of the composites. The result indicates that there are four kinds of phases in the composite, FCC-CuZnAl $(\alpha)$, BCC-CuZnAl ( $\beta)$, monoclinic martensite (M) phase and BCC-Mo. It is interesting that a small amount of $\alpha$-CuZnAl exists in the composites. The $\alpha$-CuZnAl still existed when increasing quenching temperature to $900^{\circ} \mathrm{C}$. The previous research [12] indicated only M-CuZnAl phase formed in pure CuZnAl with same composition after same heat treatment process. Mo fibers affected the phase transition of CuZnAl matrix during heat treatment. The 2-D high-energy x-ray diffraction pattern in figure $2 \mathrm{~b}$ demonstrated that the Mo fibers are strongly textured crystal with [110] direction, $\alpha-\mathrm{CuZnAl}$ is strongly textured crystal with [111] direction and $\beta$-CuZnAl is strongly textured crystal with [110] direction oriented along the wire's axis.
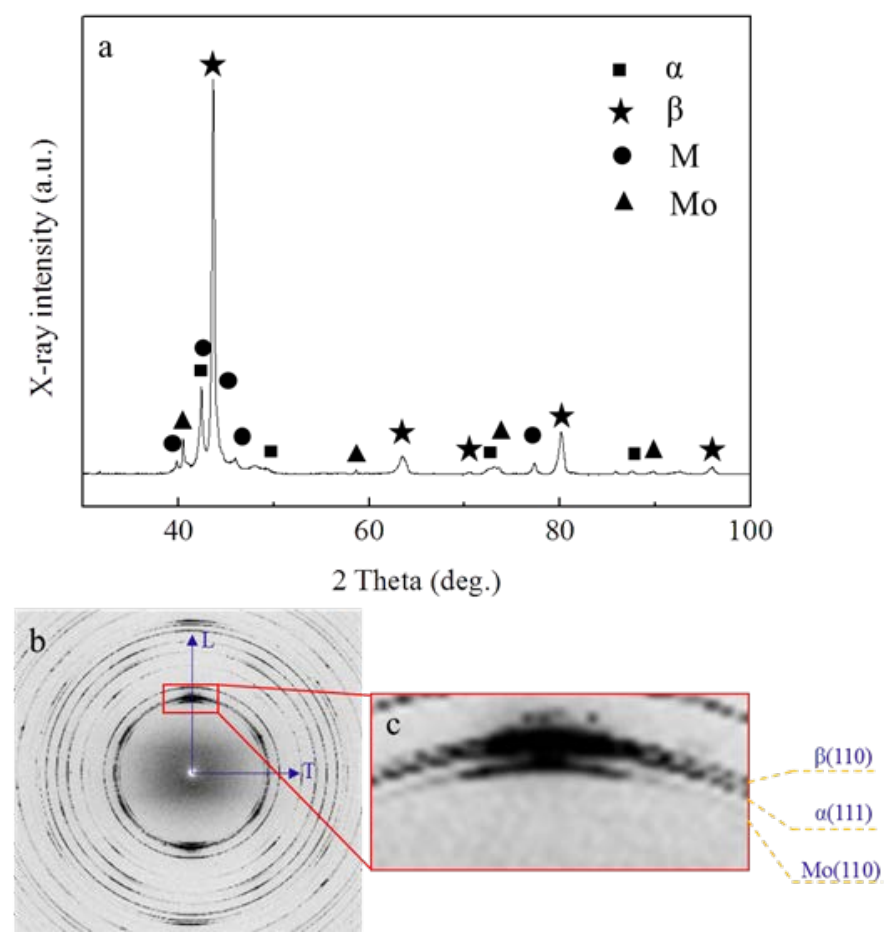

Figure 2. a. X-ray diffraction pattern of Mo fiber reinforced CuZnAl composite, b. 2-D high-energy x-ray diffraction pattern of the composite wire, the longitudinal L, and transverse $\mathrm{T}$ directions of the composite wire are labelled, c. corresponding magnification of red box.

For comparison, a CuZnAl alloy was also subjected to the same process with the Mo fiber reinforced CuZnAl composite, and the as-received composite and CuZnAl were tested by tensile machine. Figure 3a shows the stress-strain curves of the CuZnAl sample and the Mo-CuZnAl composite sample. The yield strength of the CuZnAl alloy is $230 \mathrm{MPa}$ and that of the Mo-CuZnAl composite is about 550MPa. Plasticity of the Mo-CuZnAl composite is $12 \%$ and is comparable with that of the CuZnAl sample. In situ high energy synchrotron $\mathrm{x}$-ray 
diffraction was performed to monitor deformation behaviour of different phases in the composite during tensile deformation. Figure 3b shows the evolutions of diffraction peaks of different phases in the axial direction during tensile deformation.
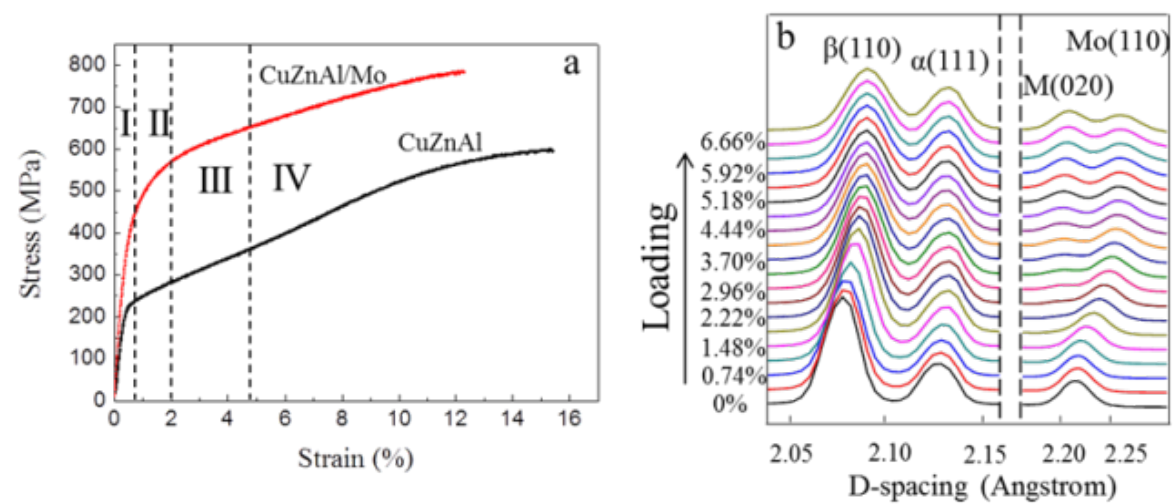

Figure 3. a. the stress-strain curves of pure CuZnAl and Mo fiber reinforced CuZnAl composite, b. the evolutions of diffraction peaks of different phases in the axial direction during tensile deformation.

In order to better characterize the load transfer and phase stress partition of the Mo fiber and the CuZnAl matrix during loading, plots of lattice strain for the Mo (110), CuZnAl- $\alpha$ (111), $\beta$ (110) and M (020) planes perpendicular to the loading direction versus the macroscopic strain are shown in Figure 4a. The lattice strain was calculated using $\left(d_{h k l}-d_{h k l}{ }^{0}\right) / d_{h k l}$, where $d_{h k l}{ }^{0}$ is the peak position at zero applied stress. It is seen that the lattice strain of Mo (110) increased continuously with increasing macroscopic strain at above $0.8 \%$, indicating continued elastic deformation. The lattice strain of Mo fiber reached a maximum of $1.8 \%$ at $4.8 \%$ of macroscopic strain, remained almost constant thereafter, implying plastic deformation. The lattice strain of $\beta$-CuZnAl reached a maximum of $0.6 \%$ at $3.5 \%$ of macroscopic strain, and the lattice strain of $\alpha-\mathrm{CuZnAl}$ reached a maximum of $0.3 \%$ at $3 \%$ of macroscopic strain.

The lattice strains of the phases are direct indications of the elastic stresses they share in the composite. The phase stresses, thus the load partition between the CuZnAl matrix and the Mo fiber during loading, are estimated according to Hooke's law as:

$$
\sigma_{M o}=E_{\langle 110\rangle, M o} \varepsilon_{\langle 110\rangle, M o}
$$

where $E_{\langle 110\rangle, M o} \approx 306 G P a$ is the Young's modulus of the $\langle 110\rangle$ oriented Mo fiber, and $\varepsilon_{\langle 110\rangle, M o}$ is the lattice d-spacing strain of Mo (110) planes orientated in the direction perpendicular to the loading direction. During loading, $\beta$-CuZnAl transformed into M-CuZnAl because of SIMT. Volume fractions of beta and M were changed, which is difficult to be used in the rule of mixture. In order to simplify calculation, we did not consider stress partitioning along the alpha, beta and M-CuZnAl. The stress partition between $\mathrm{CuZnAl}$ matrix and Mo fiber was researched. According to the rule of mixture, the phase stress of the CuZnAl matrix can be estimated from: 


$$
\sigma_{\text {appl }}=V_{\text {Mo }} \sigma_{\text {Mo }}+V_{\text {CuZnAl }} \sigma_{\text {CuZnAl }}
$$

The phase stress in the CuZnAl matrix, $\sigma_{\text {CuZnAl }}$, can therefore be estimated by the following equation:

$$
\sigma_{\text {CuZnAl }}=\left(\sigma_{\text {appl }}-V_{\text {Mo }} \sigma_{\text {Mo }}\right) / V_{\text {CuZnAl }}
$$

where $\sigma_{\text {appl }}$ is the external applied stress and $V_{M o}$ and $V_{C u Z n A l}$ are the volume fractions of the Mo fiber and the CuZnAl matrix, respectively. We define $V_{\text {phase }} \sigma_{\text {phase }} / \sigma_{\text {appl }}$ as the stress fraction carried by each phase, where $\sigma_{\text {phase }}$ and $V_{\text {phase }}$ are the phase stress and volume fraction of each phase, respectively. The calculated stress fractions carried by the Mo fiber and the CuZnAl matrix as a function of macroscopic strain upon tensile loading are shown in Figure $4 \mathrm{~b}$.
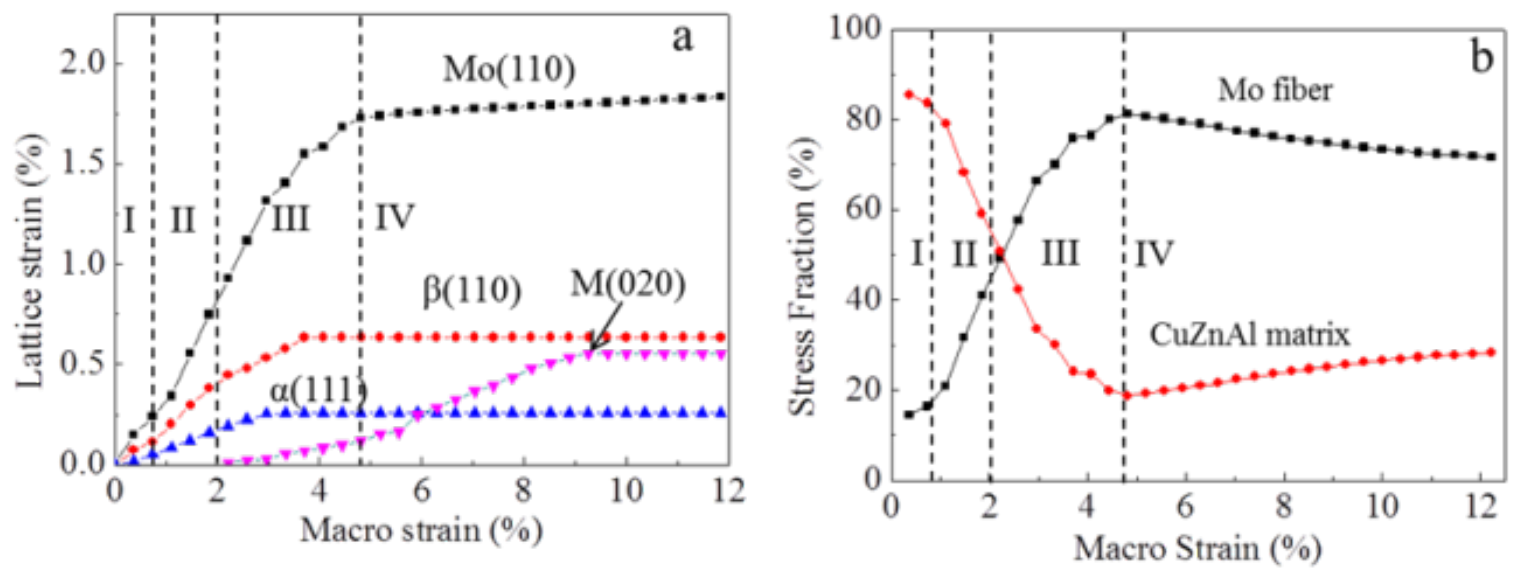

Figure 4. a. plots of lattice strain for the Mo (110), CuZnAl- $\alpha$ (111), $\beta$ (110) and M (020) perpendicular to the loading direction versus macroscopic strain, b. stress fractions carried by the Mo fiber and the CuZnAl matrix as a function of macroscopic strain upon tensile loading.

According to figures 3 and 4, the deformation process of the composite can be divided into four stages, as indicated in figure 3a. Stage I is the elastic regime, in which both the Mo fiber and the CuZnAl matrix deformed elastically. During this stage, the Mo (110) and $\beta$-CuZnAl (110) diffraction peaks shifted progressively to larger d-spacing values, as shown in figure 3b, implying elastic deformation. In stage II, the Mo fiber continued to deform elastically, as evidenced by the continuous shift of Mo (110) diffraction peak to higher d-spacing values. However, the CuZnAl matrix deformed plastically, as evident in figure 3a. In stage III, the CuZnAl matrix underwent stress induced martensite transformation. It is evidenced by the decrease of intensity of $\beta$-CuZnAl (110) diffraction peak and the increase of intensity of M-CuZnAl (020) diffraction peak. The Mo fiber continued to deform elastically as indicated by the continuous shift of Mo (110) diffraction peak towards larger d-spacing. The intensity 
of $\beta$-CuZnAl (110), $\alpha$-CuZnAl (111) and Mo (110) diffraction peak diffraction peaks remained relative constant in stage IV, implying the Mo fiber and the CuZnAl matrix deformed plastically.

It is seen that in stage I, the stress fraction carried by the matrix and the Mo fiber was kept almost constant. The stress fraction carried by the Mo fiber increased rapidly in stages II and III, within which the stress fraction carried by the matrix decreased reciprocally. This implies significant stress transfer from the CuZnAl matrix to the Mo fiber in stages II and III. In stage III, the CuZnAl matrix underwent stress induced martensitic transformation (SIMT). Mo fibers sustained increasing stress fraction during this stage. Mo fibers (10\%) in the composite bore $80 \%$ of the stress, which implied a component stress of $550 \mathrm{MPa}$ on the fibers, indicating that the Mo fibers significantly strengthened the CuZnAl matrix composites.

In stage IV, the stress fraction of the Mo fiber decreased slightly with increasing macroscopic strain, whist that of the matrix increased accordingly, implying stress transfer from the Mo fiber to the CuZnAl matrix. This is attributed to the strain hardening of the CuZnAl matrix during plastic deformation.

Many studies [13-15] indicated that, beyond the initial elastic deformation, the stress fraction carried by the reinforcement increases and the stress taken by the matrix also increases or keeps almost constant with increasing macroscopic strain. Our results are different from that. We estimated that the CuZnAl matrix underwent stress induced martensitic transformation (SIMT). The instantaneous phase transformation strain occurred in CuZnAl matrix, and did not need to increase external stress. The increasing external stress is borne mainly by the Mo fibers during this stage. We conjectured the change of modulus caused by SIMT strain resulted in the phase stress partition different from researches previously.

\section{Conclusions}

In conclusion, Mo fiber reinforced CuZnAl composite was fabricated by friction stir processing (FSP) and wire drawing. Compare to pure CuZnAl, composites have higher strength and appropriate plasticity. The maximum elastic strain of the Mo fiber achieved was $1.8 \%$, implying a component stress of $550 \mathrm{MPa}$ on the fibers. The Mo fibers, with a volume fraction of $10 \%$, carried $80 \%$ of load fraction during tensile deformation. The change of modulus caused by SIMT strain resulted in redistribution of the phase stress partition between Mo fibers and CuZnAl matrix.

\section{Acknowledgments}

This work was financially supported by the National Natural Science Foundation of China (Grant Nos. 51101170, 51231008, 51101155 and 51471028), the National 973 programs of China (2012CB619403), the Key Project of Chinese Ministry of Education (313055), Australian Research council (Grant No. DP140103805) and the Beijing Nova program (Grant No. Z141103001814039).

\section{References}


[1] Z.G. Wei and D.Z. Yang, Mater. Lett., 27 (1996), pp. 171-175

[2] C.Y. Chung, C.W.H. Lam, and S.S. Tan, Mater. Lett., 33 (1998), pp. 291-296

[3] F.d.C. Bubani, M. Sade, and F. Lovey, Mater. Sci. Eng. A, 543 (2012), pp. 88-95

[4] F. de Castro Bubani, M. Sade, and F. Lovey, Mater. Sci. Eng. A, 577 (2013), pp.147-157

[5] E.T. Thostenson, C. Li, and T.-W. Chou, Compos. Sci. Technol., 65 (2005), pp. 491-516

[6] J. Han, L. Fang, J. Sun et al., J. Appl. Phys., 112 (2012), pp. 114314

[7] P. Podsiadlo, A.K. Kaushik, E.M. Arruda et al., Science, 318 (2007), pp. 80-83

[8] Y. Dzenis, Science, 319 (2008), pp. 419-420

[9] J.N. Coleman, U. Khan, and Y.K. Gun'ko, Adv. Mater., 18 (2006), pp. 689-706

[10] S.J. Hao, D.Q. Jiang, L.S. Cui et al., Appl. Phys. Lett., 99 (2011), pp. 084103

[11] S. Hao, L. Cui, D. Jiang et al., Science, 339 (2013), pp. 1191-1194

[12] M. Franz and E. Hornbogen, Mater. Sci. Eng. A, 252 (1998), pp. 157-165

[13] Z. Li and S. Schmauder, Comput. Mater. Sci., 18 (2000), pp. 295-302

[14] M.L. Young, J. DeFouw, J.D. Almer et al., Acta Mater., 55 (2007), pp. 3467-3478

[15] P.J. Withers and A.P. Clarke, Acta Mater., 46 (1998), pp. 6585-6598 